\title{
HIV-Associated Vitiligo Totalis with Minimal Repigmentation and Alopecia Areata Diffusa During Immune-Reconstitution
}

\author{
Jason E. Sack ${ }^{*}, 1$, Salinee Rojhirunsakool ${ }^{1}$, Jag Bhawan ${ }^{1,2}$ and Thomas M. Rünger ${ }^{1}$ \\ ${ }^{1}$ Department of Dermatology, Boston University School of Medicine, USA \\ ${ }^{2}$ Dermatopathology Section, Department of Dermatology, Boston University School of Medicine, USA
}

\begin{abstract}
Background: Cutaneous findings in the setting of HIV infection encompass a broad spectrum of diseases. Only few cases of vitiligo or alopecia areata have been described in HIV/AIDS patients and it remains unclear whether there is a causal relationship between HIV/AIDS and these two conditions.

Observations: Our patient initially presented with diffuse generalized pruritic hypo- and depigmented macules and patches. She was diagnosed with advanced HIV/AIDS at that time. There was progression to vitiligo totalis followed by partial repigmentation and generalized alopecia areata diffusa with immune-reconstitution.

Conclusions: This is, to our knowledge, the first case of rapidly progressing vitiligo totalis in a patient with advanced HIV/AIDS. We conclude that this, together with the observation of repigmentation during immune-reconstitution, suggests a causal relation between vitiligo and HIV/AIDS. The different time course of the also observed alopecia areata diffusa, with first manifestation during immune-reconstitution, may be due to differences in the immune-pathogenesis between vitiligo and alopecia areata.
\end{abstract}

\section{CASE REPORT}

A 50-year-old Haitian female presented with a two month history of a progressive eruption of symmetric pruritic ill-defined hypo- and depigmented macules and patches. The eruption had begun on the face and later involved the trunk and upper and lower extremities. There was no known family history of vitiligo or other autoimmune diseases. A punch biopsy from the right arm showed reduced epidermal melanin, focal lymphocytic exocytosis, absence of melanocytes (confirmed by lack of staining with Mel-5), a sparse superficial perivascular lymphocytic infiltrate and melanophages; findings consistent with vitiligo. Topical corticosteroid therapy was supplemented with narrow-band UVB phototherapy. This combination effectively controlled the pruritus but, despite a six month three times weekly course, did not halt the progressive pigment loss. The patient was diagnosed with HIV/AIDS (Initial CD4+ cells $11 / \mathrm{mm}^{3}$; viral load 39313 copies/ml). Post initiation of HAART (Emtricitabine/Tenofovir, Efavirenz), with subsequent rising number of CD4+ cells and falling HIV viral load, she continued to rapidly depigment and was completely depigmented within five months (Fig. 1). With recovery of CD4 counts to $161 / \mathrm{mm}^{3}$, small foci of repigmentation were noted (Fig. 2) and, with continued improvement of CD4 counts up to $300 / \mathrm{mm}^{3}$, additional macular repigmentation was evident. This repigmentation developed over eight months and involved the posterior upper arms, left shin and inner thighs.

\footnotetext{
*Address correspondence to this author at the Sudbury Skin Clinic, 885 Regent Street, Suite 300, Sudbury, ON P3E 5M4, Canada; Tel: 705669 0002; Fax: 705669 1771; E-mail : jasonsack55@hotmail.com
}

Nevertheless, repigmentation was minimal, involving less than $1 \%$ of the total body surface area. Despite the enormous psychological impact vitiligo presents for most patients, our patient is happy with her "new skin color" and wishes no further intervention at this time.

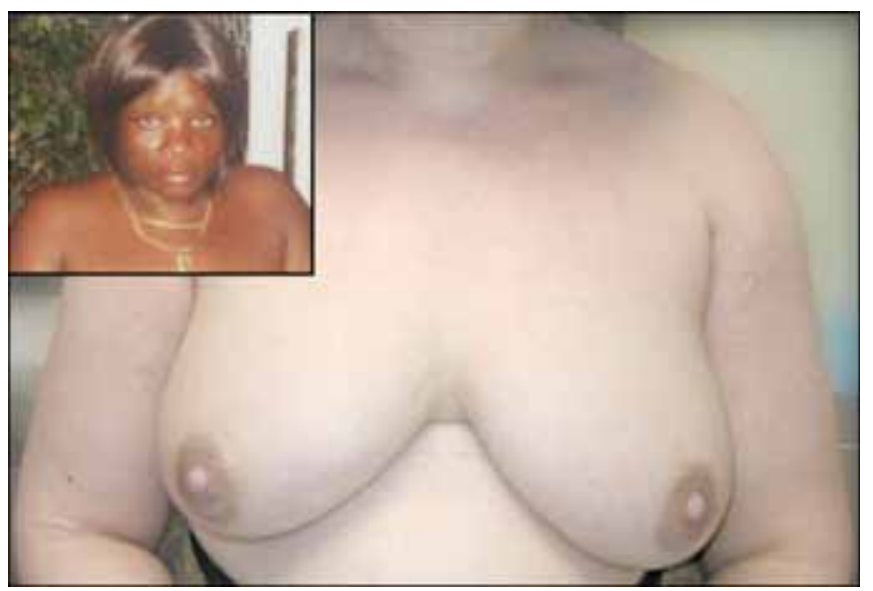

Fig. (1). Complete loss of pigmentation within nine months of first noticing hypopigmentation. The insert photograph shows the same patient (skin phototype VI) two years earlier (photograph provided by patient).

At a CD4 count of $90 / \mathrm{mm}^{3}$, the patient reported that all body hair had become white and sparse. On examination, there was thinning of scalp hair, eyebrows, eyelashes, pubic, axillary and leg hair. Infundibulae of hair follicles were preserved. All body hair had turned white with the exception of a few residual black scalp hairs. Hair loss was progressive in subsequent months (Fig. 3) without evidence of regrowth. 


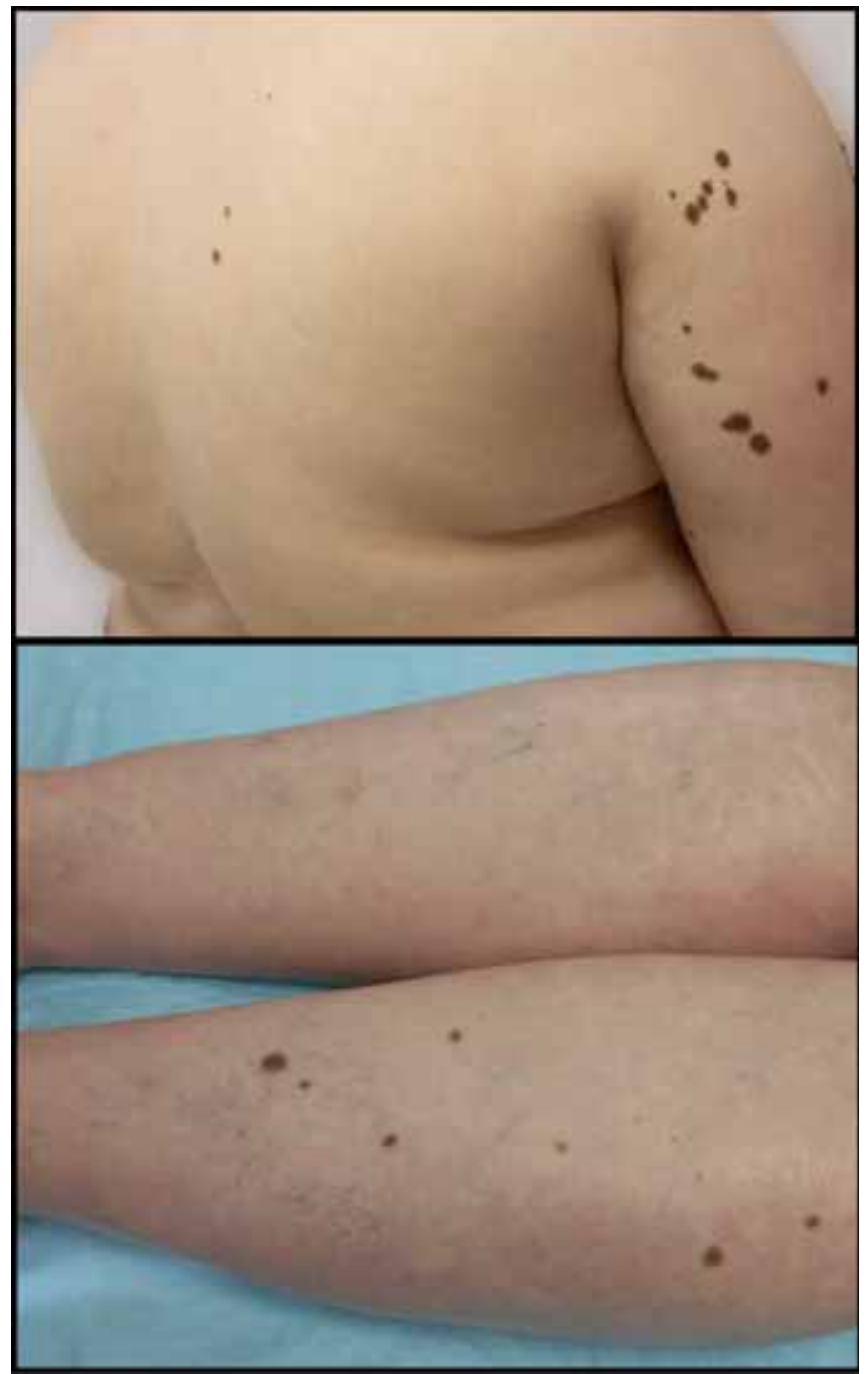

Fig. (2). Partial repigmentation during immune-reconstitution. With HAART-treatment, the CD4 count had recovered to $161 / \mathrm{mm}^{3}$ at that time.

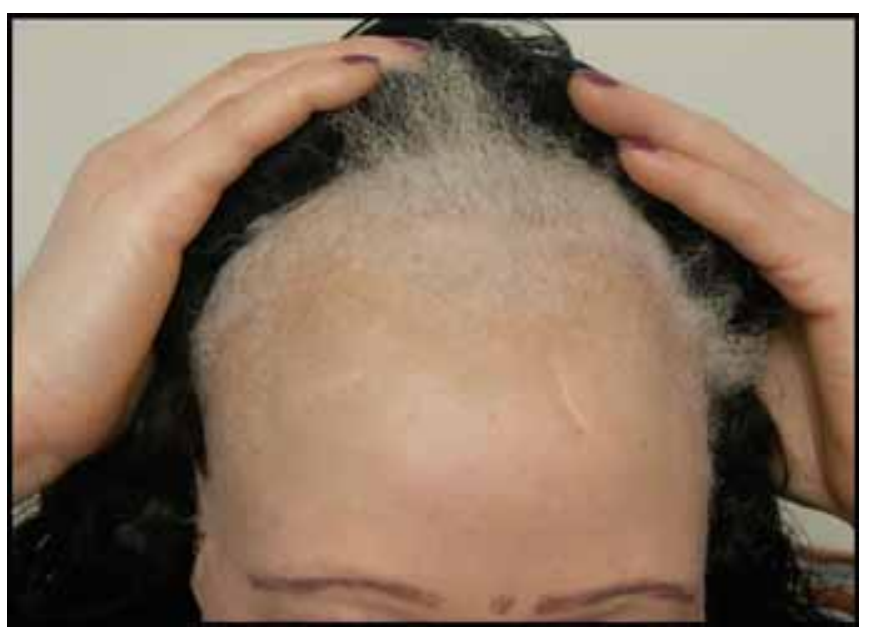

Fig. (3). Alopecia areata diffusa that developed during immunereconstitution. Patient is wearing a wig of black hair which was pulled back to reveal the extensive thinning and pigment loss with only few pigmented hairs remaining on the frontal scalp.

\section{COMMENT}

Vitiligo in a patient with HIV/AIDS was first reported by Duvic et al. in 1987 [1]. Eight more patients have been described since [2-4]. Our patient represents the first case with vitiligo totalis associated with HIV/AIDS. In addition, the rapid progression to complete depigmentation within only nine months and the initial manifestation of pigment loss at a time when the patient was severely immunocompromised $\left(11 \mathrm{CD} 4+\right.$ cells $/ \mathrm{mm}^{3}$ ) sets our case apart from the previously reported ones. Earlier reported cases had developed vitiligo sooner after HIV infection [2] but showed no progression with advanced disease. Partial repigmentation with immunereconstitution, as in our patient, has only been described once before [3].

At initial presentation, the hypo- and depigmented macules and patches were ill-defined and thus were not characteristic of typical vitiligo vulgaris. Although we cannot exclude that the depigmentation in our patient is mediated by a process different from that in vitiligo vulgaris, the histopathologic findings were supportive.

Our patient has demonstrated remarkable psychological adaptation to her skin condition by "liking her new skin color". Significant further repigmentation might prove problematic. However, we believe that generalized prominent repigmentation is unlikely given the complete loss of hair pigment indicating a lack of a residual pool of viable melanocytes to repopulate the epidermis.

The pathogenesis of melanocyte loss in vitiligo is not completely understood but an autoimmune reaction against melanocytes is likely involved [5, 6]. Several mechanisms have been suggested to explain how HIV-induced immunodeficiency can result in vitiligo [1]. Furthermore, other autoimmune phenomena, e.g. psoriasis, are well known to be associated with a declining CD4 count in HIV-patients. Unlike psoriasis, which usually clears with depletion of virtually all CD4+ T-cells, our case, where we observed disease onset and progression at that time $\left(11 \mathrm{CD} 4+\right.$ cells $\left./ \mathrm{mm}^{3}\right)$, demonstrates that CD4+ cells are not directly causing the immune-mediated killing of melanocytes. Perhaps a not yet identified infectious agent to which HIV/AIDS patients would be more susceptible is directly resulting in melanocyte death.

Anthony et al. [3] raised the possibility that vitiligo in HIV/AIDS patients may simply be coincidental but noted that all previous cases of vitiligo developed post HIVinfection. However, there is likely a reporting bias in this observation as cases with vitiligo predating HIV-infection are unlikely to be documented. Our observation of a very rapidly progressive vitiligo at the time of virtual absence of $\mathrm{CD} 4+\mathrm{T}$ cells and then repigmentation with immunereconstitution does support the hypothesis that HIV/AIDS promotes the development of vitiligo. Epidemiologic studies to investigate whether HIV/AIDS patients have a higher incidence of vitiligo may be a good tool to further substantiate this hypothesis. The significant clinical relevance would be that patients presenting with vitiligo should possibly be tested for HIV infection. Based on our case, we would suggest at least testing patients in which there are unusual manifestations or rapid progression of vitiligo. 
While an association of alopecia areata and vitiligo is widely accepted [7], there are only five patients reported with alopecia areata and HIV/AIDS [2]. Two additional HIV/AIDS patients had alopecia areata and vitiligo like our patient [2, 3]. However, the extent and the type of alopecia areata (diffusa type) in our patient is different from all other reported cases of alopecia areata in HIV/AIDS patients. While alopecia areata most commonly affects pigmented hair, often leaving only depigmented hair, we propose that the hair depigmentation in our patient is more likely a result of the vitiligo process as the hair depigmentation predated the hair loss.

Although the pathogenesis of hair follicle destruction in alopecia areata is still a matter of debate, it is believed, similar to vitiligo, to involve a T-cell-mediated autoimmune response $[6,8]$. The different time course of vitiligo and alopecia areata in our patient, with vitiligo occurring predominately before immune-reconstitution and alopecia areata afterwards, suggests that these two conditions may not only differ in their immunogenic target but also in the type of immune response.

\section{REFERENCES}

[1] Duvic M, Rapini R, Hoots WK, Mansell PW. Human immunodeficiency virus-associated vitiligo: Expression of autoimmunity with immunodeficiency? J Am Acad Dermatol 1987; 17: 656-62.

[2] Cho M, Cohen PR, Duvic M. Vitiligo and alopecia areata in patients with human immunodeficiency virus infection. South Med J 1995; 88: 489-91.

[3] Antony FC, Marsden RA. Vitiligo in association with human immunodeficiency virus infection. J Eur Acad Dermatol Venereol 2003; 17: 456-8.

[4] McGowan JW, Long JB, Johnson CA, Lynn A. Disseminated vitiligo associated with AIDS. Cutis 2006; 77: 169-73.

[5] Le Poole I, Wankowicz-Kalinska A, van den Wijngaard RM, Nickoloff BJ, Das PK. Autoimmune aspects of depigmentation in vitiligo. J Invest Dermatol Symp Proc 2004; 9: 68-72.

[6] Passeron T, Ortonne JP. Physiopathology and genetics of vitiligo. J Autoimmun 2005; 25(Suppl): 63-8.

[7] Madani S, Shapiro J. Alopecia areata update. J Am Acad Dermatol 2000; 42: 549-66.

[8] Gilhar A, Kalish RS. Alopecia areata: a tissue specific autoimmune disease of the hair follicle. Autoimmun Rev 2006; 5: 64-9. 